\title{
The BDNF p.Val66Met polymorphism, childhood trauma, and brain volumes in adolescents with alcohol abuse
}

\author{
Shareefa Dalvie ${ }^{1 *}$, Dan J Stein², Karestan Koenen ${ }^{3}$, Valerie Cardenas ${ }^{4}$, Natalie L Cuzen ${ }^{2}$, Raj Ramesar ${ }^{1}$, \\ George Fein ${ }^{4}$ and Samantha J Brooks ${ }^{2}$
}

\begin{abstract}
Background: Previous studies have indicated that early life adversity, genetic factors and alcohol dependence are associated with reduced brain volume in adolescents. However, data on the interactive effects of early life adversity, genetic factors (e.g. p.Met66 allele of BDNF), and alcohol dependence, on brain structure in adolescents is limited. We examined whether the BDNF p.Val66Met polymorphism interacts with childhood trauma to predict alterations in brain volume in adolescents with alcohol use disorders (AUDs).

Methods: We examined 160 participants (80 adolescents with DSM-IV AUD and 80 age- and gender-matched controls) who were assessed for trauma using the Childhood Trauma Questionnaire (CTQ). Magnetic resonance images were acquired for a subset of the cohort (58 AUD and 58 controls) and volumes of global and regional structures were estimated using voxel-based morphometry (VBM). Samples were genotyped for the p.Val66Met polymorphism using the TaqMan ${ }^{\circledast}$ Assay. Analysis of covariance (ANCOVA) and post-hoc t-tests were conducted using SPM8 VBM.
\end{abstract}

Results: No significant associations, corrected for multiple comparisons, were found between the BDNF p.Val66Met polymorphism, brain volumes and AUD in adolescents with childhood trauma.

Conclusions: These preliminary findings suggest that the BDNF p.Met66 allele and childhood trauma may not be associated with reduced structural volumes in AUD. Other genetic contributors should be investigated in future studies.

Keywords: Childhood trauma questionnaire, Alcohol use disorders, Magnetic resonance imaging, Voxel-based morphometry

\section{Background}

Early brain development is strongly influenced by a range of environmental factors, including exposure to substances such as alcohol [1], as well as exposure to traumas such as early adversity, abuse and neglect [2]. Neuroimaging studies have indicated that various brain regions are altered in those who are alcohol dependent. In comparison to healthy controls, individuals who are alcohol dependent have smaller prefrontal cortical volumes [3], right hippocampal volumes [4,5], amygdala [6] and grey

\footnotetext{
* Correspondence: dlvsha006@myuct.ac.za

'MRC/UCT Human Genetics Research Unit, Division of Human Genetics, Institute of Infectious Disease and Molecular Medicine, Faculty of Health Sciences, University of Cape Town, Observatory 7925, Cape Town, South Africa Full list of author information is available at the end of the article
}

and white matter volumes $[7,8]$. Studies of alcohol exposure in adolescents have, however, been confounded by drug and psychiatric comorbidity. In contrast to studies in adults, we found that in adolescents with alcohol exposure but with no comorbidity, the pattern of grey matter density differences compared to controls was limited to regions in the left lateral frontal, parietal and temporal lobes [8].

Studies of exposure to childhood trauma have been associated with reduced brain volumes in the prefrontal cortex [9] and the hippocampus [10-12]. This is consistent with animal studies, which demonstrated that rats exposed to early life stress have alterations in hippocampal volume, possibly as a result of a decreased rate of synaptic development [13]. A more recent study found that early 
life stress was associated with changes in neuronal firing rates in the hippocampal and thalamocortical brain regions in rats [14]. In a further analysis of our data on adolescents, we found that childhood trauma is associated with smaller left hippocampal and right precentral gyrus volumes in adolescents with exposure to alcohol [15].

Brain development is, however, influenced not only by exposure to alcohol and childhood stressors, but also by genetic factors. Twin studies have shown that brain structure has considerable heritability [16-18]. However, exactly which genes are responsible for brain volume variation is not yet clarified. A strong candidate is the brain derived neurotrophic factor (BDNF) gene (chr11p13) which has previously been associated with variation in brain volumes [19-21]. Of particular interest is the $p$. Val66Met functional polymorphism, which is characterized by a valine to methionine substitution at codon 66 and is located in the pro-domain of the protein [22]. Although the pro-domain is cleaved from the mature protein, it is thought to be involved in the trafficking of BDNF and specifically, p.Met66 allele carriers (heterozygous p.Val66Met and homozygous p.Met66) have lower serum BDNF levels [23], as well as smaller hippocampal and prefrontal cortical volumes [19] compared to individuals who are homozygous for the p.Val66 allele.

We hypothesized that neural development during adolescence is jointly influenced by genetic and environmental factors, such as exposure to childhood trauma or excess alcohol use. For example, those with the $B D N F$ p.Met66 allele might be more vulnerable to the effects of early life adversity and alcohol use. Based on our previous research we have found that left lateral frontal, parietal and temporal lobes as well as hippocampal volumes may be associated with adolescent alcoholism, childhood trauma, or both $[8,15]$. However, it is not yet known whether the $B D N F$ p.Val66Met polymorphism interacts with brain volume in these regions. Therefore, the aim of this investigation was to determine whether the BDNF p.Val66Met polymorphism interacts with levels of childhood trauma, and alcohol use disorders (AUD) to result in alterations in brain volume in a cohort of adolescents.

\section{Methods}

\section{Participants}

Ethical approval for this study was obtained from the Research Ethics Committees of Stellenbosch University (N06/07/128) and the University of Cape Town (HREC REF 023/2012). Detailed accounts of the procedures involved in participant recruitment are outlined in previous studies [8,24]. Briefly, subjects were English- and Afrikaans-speaking adolescents from 19 schools within the Cape Flats region (within a $30 \mathrm{~km}$ radius of the test site at Tygerberg Hospital) of the greater Cape Town metropole and were from moderately low socioeconomic backgrounds. Participants were recruited to the study by means of oral presentations at schools and advertisement via word-of-mouth. Individuals who consumed alcohol on a regular basis and those who did not consume alcohol were invited to participate in the study. Convenience sampling procedures were used. Alcohol users were selected on a first come basis from the 890 volunteers who expressed interest in being included in the study. Controls were matched for each of these individuals based on similarity of sociodemographic profile (i.e. age within 1 year; same sex, language, ethnicity, and socioeconomic status). Volunteers not meeting eligibility criteria for possible inclusion in the alcohol or control groups were excluded at the prescreening stage.

Exclusion criteria for study participation were intellectual disability, lifetime DSM-IV Axis I diagnosis other than AUD, current use of sedative or psychotropic medication, signs or history of fetal alcohol syndrome or malnutrition, sensory impairment, history of traumatic brain injury, presence of diseases that may affect the CNS (e.g. meningitis, epilepsy, HIV), less than 6 years of formal education, and lack of proficiency in English or Afrikaans. An additional exclusion criterion was lifetime dosages exceeding 30 cannabis joints or 3 methamphetamine doses. A previous study defined significant marijuana use as greater than 100 episodes [25] and another study investigated adolescent methamphetamine and cannabis users that had an average of 1099 lifetime cannabis doses and 579 (methamphetamine only) and 837 (methamphetamine and cannabis) doses [26]. Our cohort is well below those limits so we can conclude that our subjects were not significant cannabis or methamphetamine users. The final cohort consisted of 80 adolescents with DSM-IV AUD and 80 age- and gender-matched light/non-drinking controls $(\mathrm{HC})$. Blood samples were collected from each participant with the appropriate written informed assent and written informed consent was obtained from their parents or guardians.

\section{Measures \\ Early adversity}

Childhood adversity was measured by the 28 item Childhood Trauma Questionnaire- Short Form (CTQ-SF) [27]. This self-report questionnaire consists of five subscales, each measuring a specific dimension of childhood maltreatment: physical abuse, sexual abuse, emotional abuse, physical neglect and emotional neglect. Each dimension consists of 5 items [27].

\section{Alcohol use}

To determine current and past psychiatric diagnoses, each of the participants was interviewed with the Schedule for Affective Disorders and Schizophrenia for school-aged 
children (6-18 years) Lifetime Version (K-SADS-PL) [28]. In addition to the K-SADS-PL, the Timeline Followback (TLFB) procedure was used to determine lifetime history of alcohol use and drinking patterns [29].

\section{Neuroimaging \\ MRI acquisition}

Magnetic resonance images (MRIs) were collected for a subset of the cohort (58 AUD and $58 \mathrm{HC}$ ) with a $3 \mathrm{~T}$ Siemens Magnetom Allegra MR Headscanner using Syngo MR software (Siemens Medical Solutions). The scanner is located in the Cape Universities Brain Imaging Center at the Stellenbosch University Health Sciences Campus, South Africa. Images for 50 subjects $(25 \mathrm{HC}$ and 25 AUD) were acquired using a transaxial T1-weighted acquisition $(\mathrm{TR}=2080 \mathrm{~ms}$, $\mathrm{TE}=4.88 \mathrm{~mm}$, acquisition matrix $=256 \times 192)$ at $1.0 \mathrm{~mm}$ thickness. The initial review of these images revealed undesirable presence of bloodvessels in the imaging, resulting from the fact the scanner used is a head-only model. This does not allow proper saturation of the blood to suppress its signal before it enters the head. To reduce the signal from unsaturated blood, the use of a sagittal T1 protocol was instituted $(\mathrm{TR}=2200 \mathrm{~ms}, \mathrm{TE}=5.16 \mathrm{~ms}$, acquisition matrix $256 \times 256)$ at $1.0 \mathrm{~mm}$ thickness. A total of 66 individuals $(33 \mathrm{HC}$ and 33 AUD) had an MRI using the sagittal protocol only. Of the 50 individuals with a transaxial T1-weighted acquisition, 25 individuals (9 $\mathrm{HC}$ and 16 AUD) had an additional MRI with the sagittal protocol. In prior work we demonstrated that the two acquisition protocols produced comparable images that could be combined for analysis [8], although out of an abundance of caution we match the HC and AUD subjects on imaging protocol in imaging analyses reported in this paper.

\section{MRI analysis}

After manually reorienting and realigning the cross-hair on the AC-PC plane in all our nifti-converted DICOM $\mathrm{T} 1$ images, and imposing initial quality control for signal artifacts, morphological changes were calculated using the voxel-based morphometry (VBM) default unified segmentation approach [30] in SPM8 (http://www.fil.ion. ucl.ac.uk/spm/software/spm8/). First we co-registered the T1 images to the Cincinnati Children's Hospital global old children template (www. irc.cchmc.org/software/ pedbrain.php). Using the old children templates for grey matter, white matter and cerebrospinal fluid, our images were then classified under the segmentation step as grey matter (GM), white matter (WM), and cerebrospinal fluid (CSF) and maps of GM probability at each voxel were derived. The GM probability maps were spatially normalized and co-registered using the same segmented template. The probability maps of gray matter were "modulated" to account for the effect of spatial normalisation, by multiplying the probability value of each voxel by its relative volume in native space before and after warping. Modulated images were smoothed with an $8 \mathrm{~mm}$ 'Full Width Half Maximum [FWHM]' Gaussian kernel, in line with other recent VBM studies. This smoothing kernel was applied prior to statistical analysis, to reduce signal noise and to correct for image variability unaccounted for by spatial normalization. All subsequent VBM analyses were corrected for total matter volume (TMV), representing total GM and WM but not CSF, as there is some evidence that CSF is over-estimated by SPM8 [31].

\section{Genotyping}

DNA from subjects and controls was extracted using the Maxwell $^{\circ} 16$ Blood DNA purification kit (AS1010) (Promega) using the Maxwell 16 instrument (Promega) at the Centre for Proteomic and Genomic Research (CPGR) (Cape Town, South Africa). Genotyping was performed using the TaqMan ${ }^{\bullet}$ SNP Genotyping Assay, which was run on the AB7900HT qPCR instrument (Life Technologies) at the CPGR (Cape Town, South Africa). Assay quality control measures included a no template control and a positive control. Genotyping data was validated using an Illumina Infinium iSelect custom 6000 bead chip which was run on Illumina's BeadStation 500G Systems at the University of Michigan DNA Sequencing Core (Michigan, USA).

\section{Statistical analysis}

The Shapiro-Wilk test was used to determine whether the continuous variables were normally distributed. The Mann Whitney U-test was performed to determine whether there were differences in these variables between the genders and the groups (HC vs AUD). Genotype by group (AUD vs $\mathrm{HC}$ ) association calculations were performed using logistic regression using the total cohort of $80 \mathrm{HC}$ and 80 AUD. All of the above mentioned tests were carried out using the statistical program SPSS [32]. Using the Exact test, deviation from Hardy-Weinberg Equilibrium (HWE) was calculated using the genetics package in the statistical environment $\mathrm{R}$ (http://r.adu.org.za/).

In the smaller imaging cohort of $58 \mathrm{HC}$ and $58 \mathrm{AUD}$, to examine the main effects of group (AUD and HC), CTQ score (high vs low), genotype (homozygous p.Val66 vs. p. Val66Met and homozygous p.Met66) and the interaction between genotype and CTQ on brain volume data, $2 \times 2$ ANCOVA using VBM in the SPM8 package (http:http:// www.fil.ion.ucl.ac.uk/spm/software/spm8/) was implemented. Based on our previous findings, region of interest (ROI) analyses were derived from templates in the aal atlas, representing the left lateral frontal, parietal and temporal lobes as well as hippocampal volumes $[8,15]$. AUD and $\mathrm{HC}$ subjects were matched in terms of age, gender, 
and protocol. Age and TMV was added as covariates of no interest to control for global differences in head size and emphasise local volume differences, and total CTQ score was included as a covariate of interest. All statistical analyses were corrected for multiple comparisons at the peak voxel level using the family-wise error (FWE).

\section{Results}

\section{Demographics, alcohol use, trauma and genotype} information

Sociodemographic and clinical data for the cohort are presented in Table 1. As expected, adolescents with AUDs had significantly higher lifetime doses of alcohol than the HC group (Mann Whitney U-test $p$-value $<0.001$; Table 2). In the total cohort, the median total CTQ score was 36.50 and 42.00 for the HC and AUD group, respectively. This difference in median total CTQ scores was statistically significant (Mann Whitney U-test $p$-value $=0.023)$ (see Table 2 for further details, including the subscale scores). The median ages of the HC and AUD groups for the total cohort were 14.92 and 14.94, respectively (Table 1). The majority of the study participants were Afrikaans speaking and the median number of years of education was 8.0 years for both groups (HC and AUD). The median number of alcohol life dose units for the AUD group in the total cohort was 1125.50 , where a unit refers to one beer or wine cooler, one glass of wine, or one $43 \mathrm{~g}$ shot of liquor (on its own, or in a mixed drink).

The genotype frequencies in the total cohort ( 80 AUD and $80 \mathrm{HC}$ ) were homozygous p.Val66 $=0.79$, heterozygous p.Val66Met $=0.15$ and homozygous $\mathrm{p}$. Met66 $=0.06$ for cases and homozygous p.Val66 $=0.83$, heterozygous p.Val66Met $=0.16$ and homozygous p. Met66 $=0.01$ for controls. The genotype frequencies in the imaging cohort (58 AUD and $58 \mathrm{HC}$ ) were homozygous p.Val66 $=0.76$, heterozygous p.Val66Met $=0.17$ and homozygous p.Met $66=0.07$ for cases and homozygous p.Val66 $=0.78$, heterozygous p.Val66Met $=0.21$ and homozygous p.Met66 $=0.02$ for controls. As there were a small number of individuals with the p.Met66 allele, heterozygous individuals were grouped with individuals homozygous for the p.Met66 allele. There was no significant association between the p.Val66Met polymorphism and AUD when comparing all genotype groups

Table 1 Median values and interquartile range for cohort characteristics

\begin{tabular}{|c|c|c|c|c|c|c|}
\hline \multirow{2}{*}{$\begin{array}{l}\text { Variable } \\
\text { Total cohort }\end{array}$} & \multicolumn{3}{|l|}{$\mathrm{HC}$} & \multicolumn{3}{|l|}{ AUD } \\
\hline & $\begin{array}{l}\text { Females } \\
(n=47)\end{array}$ & Males $(n=33)$ & Total $(n=80)$ & Females $(n=47)$ & Males $(n=33)$ & Total $(n=80)$ \\
\hline Imaging cohort $^{b}$ & Females $(n=33)$ & Males $(n=25)$ & Total $(n=58)$ & Females $(n=33)$ & Males $(n=25)$ & Total $(n=58)$ \\
\hline \multirow{2}{*}{ Age (years) } & 14.89 (15.32-14.4) & $14.94(15.45-14.23)$ & $14.92(15.33-14.36)$ & 14.89 (15.59-14.31) & $14.98(15.51-14.52)$ & $14.94(15.53-14.47)$ \\
\hline & $14.71(15.14-14.35)$ & 15.07 (15.54-14.32) & $14.77(15.33-14.35)$ & $14.89(15.64-14.06)$ & $15.17(15.57-14.52)$ & $14.98(15.60-14.41)$ \\
\hline \multirow{2}{*}{ Education (years) } & $8.0(8.0-7.0)$ & $8.0(8.0-7.0)$ & $8.0(8.0-7.0)$ & $8.0(8.0-7.0)$ & $8.0(8.0-7.0)$ & $8.0(8.0-7.0)$ \\
\hline & $8.0(8.0-7.0)$ & $8.0(8.0-7.0)$ & $8.0(8.0-7.0)$ & $8.0(9.0-7.0)$ & $8.0(8.0-7.0)$ & $8.0(8.0-7.0)$ \\
\hline \multirow{2}{*}{ \% Afrikaans-speaking } & 63.8 & 78.8 & 70.0 & 63.8 & 75.8 & 68.8 \\
\hline & 69.7 & 84 & 75.9 & 60.6 & 72.0 & 65.5 \\
\hline \multirow{2}{*}{$\begin{array}{l}\text { Alcohol life dose } \\
\text { units }^{a}\end{array}$} & $1.0(9.00-0)$ & $1.0(4.0-0)$ & $1.0(5.75-0.0)$ & $1152.0(2008.0-480.0)$ & $1012.0(2076.0-396.0)$ & $1125.50(2032.0-441.0)$ \\
\hline & $1.0(9.00-0)$ & $1.0(3.5-0)$ & $1.0(4.25-0.0)$ & $892.0(1980.0-442.0)$ & $1012.0(2076.0-396.0)$ & $962.0(1987.0-429.0)$ \\
\hline \multirow{2}{*}{ CTQ-Physical Abuse } & $5.0(7.0-5.0)$ & $5.0(6.0-5.0)$ & $5.0(7.0-5.0)$ & $5.0(7.0-5.0)$ & $7.0(10.0-5.0)$ & $5.0(7.0-5.0)$ \\
\hline & $5.0(6.5-5.0)$ & $5.0(6.0-5.0)$ & $5.0(6.0-5.0)$ & $5.0(7.0-5.0)$ & $7.0(12.0-5.0)$ & $6.0(7.5-5.0)$ \\
\hline \multirow{2}{*}{ CTQ-Sexual abuse } & $5.0(5.0-5.0)$ & $5.0(5.0-5.0)$ & $5.0(5.0-5.0)$ & $5.0(6.0-5.0)$ & $6.0(9.0-5.0)$ & $5.0(7.0-5.0)$ \\
\hline & $5.0(5.0-5.0)$ & $5.0(5.0-5.0)$ & $5.0(5.0-5.0)$ & $5.0(5.0-5.0)$ & $6.0(12.0-5.0)$ & $5.0(7.0-5.0)$ \\
\hline \multirow{2}{*}{ CTQ-Emotional Abuse } & $6.0(9.0-5.0)$ & $6.0(8.0-5.0)$ & $6.0(8.75-5.0)$ & $8.0(11.0-6.0)$ & $9.0(11.5-5.0)$ & $8.0(11.0-6.0)$ \\
\hline & $5.0(8.5-5.0)$ & $6.0(8.5-5.0)$ & $6.0(8.25-5.0)$ & $8.0(11.0-6.0)$ & $9.0(11.5-5.0)$ & $8.0(11.0-6.0)$ \\
\hline \multirow{2}{*}{ CTQ-Physical Neglect } & $6.0(9.0-5.0)$ & $8.0(10.5-5.0)$ & $7.0(9.0-5.0)$ & $9.0(11.0-7.0)$ & $8.0(13.0-5.5)$ & $8.50(12.0-6.0)$ \\
\hline & $5.0(9.0-5.0)$ & $7.0(12.0-5.5)$ & $7.0(9.25-5.0)$ & $8.0(11.0-6.5)$ & $9.0(12.5-5.5)$ & $8.0(12.0-6.0)$ \\
\hline \multirow{2}{*}{$\begin{array}{l}\text { CTQ-Emotional } \\
\text { Neglect }\end{array}$} & $10.0(13.0-6.0)$ & $11.0(18.5-8.0)$ & $11.0(15.0-7.0)$ & $12.0(17.0-7.0)$ & $12.0(19.0-8.0)$ & $12.0(18.0-8.0)$ \\
\hline & $10.0(13.0-6.0)$ & $13.0(21.0-9.0)$ & $11.0(17.0-7.75)$ & $12.0(17.5-7.5)$ & $12.0(17.0-8.0)$ & $12.0(17.25-8.0)$ \\
\hline \multirow{2}{*}{ CTQ-total score } & $35.0(46.0-29.0)$ & $39.0(47.0-30.5)$ & $36.50(46.0-29.25)$ & $42.0(51.0-34.0)$ & $42.0(60.0-30.0)$ & $42.00(53.5-33.25)$ \\
\hline & $35.0(46.0-29.0)$ & $39.0(52.0-30.5)$ & $36.0(49.0-29.75)$ & $42.0(48.0-34.5)$ & $42.0(65.5-33.0)$ & $42.0(52.0-34.75)$ \\
\hline
\end{tabular}


Table 2 Comparison of cohort characteristics for HC and AUD groups- $p$-values from Mann Whitney U-test

\begin{tabular}{|c|c|c|c|c|c|}
\hline & \multirow{2}{*}{$\begin{array}{l}\text { HC } \\
\text { Females vs males }\end{array}$} & \multirow{2}{*}{$\begin{array}{l}\text { AUD } \\
\text { Females vs males }\end{array}$} & \multicolumn{3}{|c|}{ HC vs AUD } \\
\hline & & & Females & Males & Total \\
\hline \multirow{2}{*}{ Age (years) } & 0.949 & 0.581 & 0.589 & 0.401 & 0.313 \\
\hline & ${ }^{d} 0.162$ & 0.388 & 0.254 & 0.535 & 0.159 \\
\hline \multirow{2}{*}{ Education (years) } & 0.132 & 0.646 & 0.517 & 0.192 & 0.751 \\
\hline & 0.689 & 0.729 & 0.706 & 0.671 & 0.577 \\
\hline \multirow{2}{*}{$\%$ Afrikaans-speaking ${ }^{c}$} & 0.151 & 0.257 & 1.000 & 0.769 & 0.864 \\
\hline & & 0.366 & 0.438 & 0.306 & 0.221 \\
\hline \multirow{2}{*}{ Alcohol life dose units } & 0.385 & 0.503 & $<0.001$ & $<0.001$ & $<0.001$ \\
\hline & 0.538 & 0.881 & $<0.001$ & $<0.001$ & $<0.001$ \\
\hline \multirow{2}{*}{ CTQ-Physical Abuse } & 0.561 & 0.023 & 0.983 & 0.018 & 0.116 \\
\hline & 0.807 & 0.008 & 0.678 & 0.008 & 0.031 \\
\hline \multirow{2}{*}{ CTQ-Sexual abuse } & 0.455 & 0.018 & 0.398 & 0.001 & 0.004 \\
\hline & 0.729 & 0.036 & 0.615 & 0.011 & 0.030 \\
\hline \multirow{2}{*}{ CTQ-Emotional Abuse } & 0.806 & 0.640 & 0.025 & 0.074 & 0.004 \\
\hline & 0.967 & 0.950 & 0.034 & 0.063 & 0.005 \\
\hline \multirow{2}{*}{ CTQ-Physical Neglect } & 0.199 & 0.984 & 0.014 & 0.605 & 0.032 \\
\hline & 0.084 & 0.658 & 0.025 & 0.747 & 0.074 \\
\hline \multirow{2}{*}{ CTQ-Emotional Neglect } & 0.109 & 0.984 & 0.128 & 0.686 & 0.410 \\
\hline & 0.072 & 0.747 & 0.226 & 0.289 & 0.881 \\
\hline \multirow{2}{*}{ CTQ-total score } & 0.384 & 0.443 & 0.048 & 0.162 & 0.023 \\
\hline & 0.203 & 0.362 & 0.080 & 0.268 & 0.062 \\
\hline
\end{tabular}

${ }^{\mathrm{c}}$ Pearson chi-squared test $($ d.f. $=1) .{ }^{\mathrm{d}}$ Imaging cohort values are indicated in italics.

$(p$-value $=0.323)$ independently and when grouping the heterozygous p.Val66Met and homozygous p.Met66 genotypes together $(p$-value $=0.549)$. For the $\mathrm{HC}$ group, this polymorphism was in HWE $(p$-value $=0.51)$. Besides CTQ-emotional abuse $(p$-value $=0.032)$, none of the sociodemographic or CTQ scores differed between the homozygous p.Val66 group and the p.Met66 (homozygous and heterozygous) group.

\section{Voxel-based morphometry}

We conducted a $2 \times 2$ ANCOVA of brain volume differences for group (AUD, HC) and BDNF status (homozygous p.Val66 vs. heterozygous p.Val66Met and homozygous p.Met66 allele), using VBM and included CTQ total score as a covariate. We did not find any statistically significant associations between the BDNF p.Val66Met polymorphism, structural brain volumes and AUD, for main effects or as an interaction. Our strongest findings, uncorrected for multiple comparisons and therefore preliminary, was found in the left lateral prefrontal cortex (PFC) (Table 3). Uncorrected main effects of AUD status and genotype were observed in the left lateral PFC $(\mathrm{x}=-43, \mathrm{y}=48, \mathrm{z}=-17, \mathrm{p}<0.001)$, respectively. Specifically, individuals in the $\mathrm{HC}$ group, homozygous for the
p.Val66 allele, had larger left lateral PFC volumes than individuals in the AUD group with the same genotype. When only examining the AUD group, smaller left lateral PFC volume $(x=-38, y=13, z=28, p<0.001)$ was observed in individuals with the p.Met66 allele.

As an additional $2 \times 2$ ANCOVA, we examined CTQ as a main effect and as an interaction with $B D N F$ genotype, controlling for age, TMV and alcohol life dose as covariates. Thus, we examined high vs. low CTQ, dichotomized by percentile, and BDNF dichotomized by homozygous p.Val66 vs. heterozygous p.Val66Met and homozygous p.Met66. Using this approach an uncorrected main effect of CTQ was observed in the left parahippocampal gyrus $(x=-26, y=-24 \mathrm{z}=-25$, $\mathrm{p}<0.001)$. An uncorrected interaction was shown between CTQ and BDNF genotype in the fusiform gyrus $(x=39, y=-59 \quad z=-21, \quad p<0.001)$. Post-hoc t-tests (uncorrected) revealed smaller volumes in the middle occipital gyrus $(\mathrm{x}=-17, \mathrm{y}=-93 \mathrm{z}=14, \mathrm{p}<0.001)$ and middle frontal gyrus $(x=-27, y=25 z=-18, p<0.001)$, in individuals with high CTQ total scores compared to those with low scores, in individuals with the p.Met66 allele and those homozygous for the p.Val66 allele, respectively (Table 3). 
Table $32 \times 2$ ANCOVA (group $x$ genotype) matched for age, gender and protocol

\begin{tabular}{|c|c|c|c|c|c|c|c|}
\hline \multirow[b]{2}{*}{ Brain region } & \multicolumn{3}{|c|}{$\mathrm{MNI}^{\mathrm{e}}$ Coordinates } & \multirow[b]{2}{*}{ Brodmann Area } & \multirow[b]{2}{*}{ Cluster Size (Voxels) } & \multirow[b]{2}{*}{ Z-statistic } & \multirow[b]{2}{*}{ Cluster p-value } \\
\hline & $x$ & $y$ & $\mathbf{z}$ & & & & \\
\hline \multicolumn{8}{|l|}{ Ancova analyses: } \\
\hline \multicolumn{8}{|l|}{ Main effect of group } \\
\hline Left lateral prefrontal cortex & -43 & 48 & -17 & 11 & 125 & 3.64 & $<0.001$ \\
\hline \multicolumn{8}{|l|}{ Main effect of genotype } \\
\hline Left lateral prefrontal cortex & -43 & 48 & -17 & 11 & 129 & 3.66 & $<0.001$ \\
\hline Left occipital lobe & -32 & -88 & 3 & 19 & 58 & 3.52 & $<0.001$ \\
\hline \multicolumn{8}{|l|}{ Main effect of CTQ total score } \\
\hline Left parahippocampal gyrus & -26 & -24 & -25 & 35 & 958 & 4.16 & $<0.001$ \\
\hline \multicolumn{8}{|c|}{ Genotype $x$ alcohol group interaction } \\
\hline Left lateral prefrontal cortex & -43 & 48 & -17 & 11 & 32 & 3.33 & $<0.001$ \\
\hline \multicolumn{8}{|c|}{ CTQ total score $x$ genotype interaction } \\
\hline Fusiform gyrus & 39 & -59 & -21 & $N A^{f}$ & 890 & 3.69 & $<0.001$ \\
\hline \multicolumn{8}{|l|}{ POST-HOC t-tests ${ }^{\mathrm{g}}$ : } \\
\hline \multicolumn{8}{|c|}{ Genotype post-hoc t-test: Val/Nal > Val/Met + Met/Met } \\
\hline Left lateral prefrontal cortex & -40 & 12 & 28 & 9 & 29 & 3.24 & $<0.001$ \\
\hline \multicolumn{8}{|c|}{ Within-group genotype t-test (AUD): ValNal > Val/Met + Met/Met } \\
\hline Left lateral prefrontal cortex & -38 & 13 & 28 & 9 & 17 & 3.18 & $<0.001$ \\
\hline \multicolumn{8}{|c|}{ Between-group genotype t-test (ValNal): HC > AUD } \\
\hline Left lateral prefrontal cortex & -20 & 23 & -30 & 11 & 332 & 3.88 & $<0.001$ \\
\hline \multicolumn{8}{|c|}{ Within-group CTQ t-test ( Val/Met + Met/Met): low > high CTQ } \\
\hline Middle Occipital gyrus & -17 & -93 & 14 & 18 & 708 & 4.78 & $<0.001$ \\
\hline \multicolumn{8}{|c|}{ Within-group CTQ t-test (ValNal): low > high CTQ } \\
\hline Middle frontal gyrus & -27 & 25 & -18 & NA & 708 & 4.06 & $<0.001$ \\
\hline
\end{tabular}

${ }^{e} \mathrm{MNI}=$ Montreal Neurological Institute CoordinateSs. ${ }^{f}$ Not applicable. ${ }^{9}$ Besides the CTQ t-tests, all post-hoc t-tests were analysed with CTQ as a covariate of interest.

\section{Discussion}

This investigation sought to determine whether the $B D N F$ p.Val66Met is associated with brain volume alterations in a cohort of adolescents with AUD and who have experienced childhood trauma. In a previous analysis of structural brain volume in adolescents with AUD, AUD was associated with reduced brain volumes in left lateral frontal, temporal and parietal regions [8]. Additionally, in a study conducted by our group, we controlled for childhood trauma in an adolescent AUD cohort [15], and found reduced frontal and temporal cortices and hippocampal volumes. Here we attempted to extend previous work by examining whether the BDNF p.Val66Met polymorphism, AUD and childhood trauma jointly affect brain volume in adolescents. We were unable to find any significant main effects or interaction between the BDNF p.Val66Met, levels of childhood trauma, and AUD resulting in structural brain volume differences. This is consistent with previous studies which did not find an effect of this polymorphism on brain volumes and AUD [33-36].
As part of a secondary exploratory analysis, when examining findings uncorrected for multiple comparisons, the BDNF p.Met66 allele was associated with smaller lateral PFC volume, an area involved with behavioral executive functioning [37]. Similarly, previous studies have shown an association between the $B D N F$ p.Met66 allele and reduced dorsolateral PFC (DLPFC) volumes [19,38]. This preliminary and uncorrected finding is also in accordance with a previous study which found that childhood emotional neglect was associated with reduced DLPFC [39]. Most studies report associations between the p.Met66 allele and decreases in hippocampal volume $[19,20,40,41]$. This association between the p.Met66 allele and hippocampal volume is also observed in individuals with high levels of childhood trauma [42]. However, it has been proposed that adverse hippocampal development also significantly impinges on DLPFC development [43], as there is some evidence that these regions are functionally connected [44]. Thus, having the BDNF p.Val66Met polymorphism may interact negatively with the experience 
of childhood trauma, which may in turn affect the development of the hippocampus and the DLPFC [45].

One of the limitations of this study is the small sample size. An increase in sample size may effectively increase the power of the study and enable the detection of findings that are corrected for multiple comparisons. Certainly, studies with larger cohorts are needed to confirm our preliminary interpretations of the first exploration into how the BDNF p.Val66Met genotype and early life adversity interact with brain volumes in adolescents with AUD. Also, only one polymorphism within the BDNF gene was investigated. To gain a broader understanding of the role of $B D N F$ in brain volume variation, AUD and childhood adversity, variation (in linkage equilibrium) across the entire gene should be considered as well as the expression of the gene in brain volumes of interest. As the entire cohort in this study consisted of adolescents of mixed ancestry, ethnicity was not added as a covariate in the analysis. However, the mixed ancestry group is an admixed population; therefore the analysis could have been influenced by population stratification. Furthermore, no information was obtained regarding the familial history of AUDs in our subjects, a possible confounder. Previous research has shown that a positive family history of alcohol use has an association with increased alcohol consumption in college students [46]. Another limitation is the fact that brain images were obtained using different imaging protocols. However, we attempted to overcome this by matching scans based on protocol and have shown previously that images obtained from two different protocols could be combined for analysis [8].

\section{Conclusions}

In conclusion, these preliminary findings suggest that carrying the BDNF p.Met66 allele when exposed to early life adversity may not be associated with differential brain volumes in adolescents with AUD. Other genetic contributors should be investigated in future work.

\footnotetext{
Abbreviations

AUD: Alcohol Use Disorders; BDNF: Brain derived Neurotrophic Factor; CPGR: Centre for Proteomic and Genomic Research; CSF: Cerebrospinal Fluid; CTQ: Childhood Trauma Questionnaire; CTQ-SF: Childhood Trauma Questionnaire- Short Form; DLPFC: Dorsolateral Prefrontal Cortex; FWE: Family-Wise Error; GM: Grey Matter; HC: Healthy Controls; HWE: Hardy-Weinberg Equilibrium; K-SADS-PL: Schedule for Affective Disorders and Schizophrenia for school-aged children Lifetime Version; MNI: Montreal Neurological Institute; MRI: Magnetic Resonance Images; Met: Methioinine; PFC: Prefrontal Cortex; ROI: Region of Interest; TLFB: Timeline Followback; TMV: Total Matter Volume; Val: Valine; VBM: Voxel Based Morphometry; WM: White Matter.
}

\section{Competing interests}

The authors declare that they have no competing interest.

\section{Authors' contributions}

SD coordinated the molecular genetics work, performed the statistical analysis, and helped to draft the manuscript. DS conceived of the study, participated in its design and coordination and helped to draft the manuscript. KK participated in the study design and coordination. VC participated in the study design and coordination and helped to draft the manuscript. NC participated in the study design and coordination. RR conceived of the study and participated in its design and coordination. GF conceived of the study and participated in its design and coordination. SJB performed the brain imaging and statistical analysis, and helped to draft the manuscript. All authors read and approved the final manuscript.

\section{Authors' information}

SD is a PhD student at the Division of Human Genetics at the University of Cape Town. DS is the head of the Department of Psychiatry and Mental Health at the University of Cape Town. KK is a Director at the Psychiatric-Neurological Epidemiology Cluster and Professor of Epidemiology at the Mailman School of Public Health at Columbia University. VC is an investigator at the Neurobehavioral Research Inc. NC was an MSc student at the Department of Psychiatry and Mental Health at the University of Cape Town. RR is the Head of the Division of Human Genetics at the University of Cape Town. GF is the President and CEO of the Neurobehavioral Research Inc. SJB is a Postdoctoral Fellow at the Department of Psychiatry and Mental Health at the University of Cape Town.

\section{Acknowledgements}

This work was supported by an NIAAA grant (AA016303), and the Claude Leon Foundation Fellowship (SJB). The authors acknowledge the support of the National Research Foundation (NRF), the Medical Research Council of South Africa and the University of Cape Town (UCT).

\section{Author details}

${ }^{1}$ MRC/UCT Human Genetics Research Unit, Division of Human Genetics, Institute of Infectious Disease and Molecular Medicine, Faculty of Health Sciences, University of Cape Town, Observatory 7925, Cape Town, South Africa. ${ }^{2}$ Department of Psychiatry and Mental Health, University of Cape Town, Observatory, Cape Town, South Africa. ${ }^{3}$ Mailman School of Public Health, Columbia University, New York, NY, USA. ${ }^{4}$ Neurobehavioral Research Inc, Honolulu, HI, USA.

Received: 11 August 2014 Accepted: 10 November 2014 Published online: 16 December 2014

\section{References}

1. Squeglia LM, Jacobus J, Tapert SF: The influence of substance use on adolescent brain development. Clin EEG Neurosci 2009, 40(1):31-38.

2. Glaser D: Child abuse and neglect and the brain-a review. J Child Psychol Psychiatry 2000, 41(1):97-116.

3. Medina KL, McQueeny T, Nagel BJ, Hanson KL, Schweinsburg AD, Tapert SF: Prefrontal cortex volumes in adolescents with alcohol use disorders: unique gender effects. Alcohol Clin Exp Res 2008, 32(3):386-394.

4. Agartz I, Momenan R, Rawlings RR, Kerich MJ, Hommer DW: Hippocampal volume in patients with alcohol dependence. Arch Gen Psychiatry 1999 56:356-366.

5. De Bellis MD, Clark DB, Beers SR, Soloff PH, Boring AM, Hall J, Kersh A, Keshavan MS: Hippocampal volume in adolescent-onset alcohol use disorders. Am J Psychiatry 2000, 157(5):737-744.

6. Makris N, Oscar-Berman M, Jaffin SK, Hodge SM, Kennedy DN, Caviness VS: Decreased volume of the brain reward system in alcoholism. Biol Psychiatry 2008, 64(3):192-202.

7. Gazdzinski S, Durazzo TC, Studholme C, Song E, Banys P, Meyerhoff DJ: Quantitative brain MRI in alcohol dependence: preliminary evidence for effects of concurrent chronic cigarette smoking on regional brain volumes. Alcohol Clin Exp Res 2005, 29(8):1484-1495.

8. Fein G, Greenstein D, Cardenas VA, Cuzen NL, Fouche J, Ferrett $H$, Thomas K, Stein DJ: Cortical and subcortical volumes in adolescents with alcohol dependence but without substance or psychiatric comorbidities. Psychiatry Res Neuroimaging 2013, 214(1):1-8.

9. van Harmelen A, van Tol M, van Der Wee NJ, Veltman DJ, Aleman A, Spinhoven $P$, van Buchem MA, Zitman FG, Penninx BW, Elzinga BM: Reduced medial prefrontal cortex volume in adults reporting childhood emotional maltreatment. Biol Psychiatry 2010, 68(9):832-838. 
10. Vythilingam M, Heim C, Newport J, Miller AH, Anderson E, Bronen R, Brummer $M$, Staib L, Vermetten E, Charney DS: Childhood trauma associated with smaller hippocampal volume in women with major depression. Am J Psychiatry 2002, 159(12):2072-2080.

11. Dannlowski U, Stuhrmann A, Beutelmann V, Zwanzger $P$, Lenzen T, Grotegerd D, Domschke K, Hohoff C, Ohrmann P, Bauer J: Limbic scars: long-term consequences of childhood maltreatment revealed by functional and structural magnetic resonance imaging. Biol Psychiatry 2012, 71(4):286-293.

12. Samplin $E$, Ikuta T, Malhotra AK, Szeszko PR, Derosse P: Sex differences in resilience to childhood maltreatment: effects of trauma history on hippocampal volume, general cognition and subclinical psychosis in healthy adults. J Psychiatr Res 2013, 47:1174-1179.

13. Andersen SL, Teicher MH: Delayed effects of early stress on hippocampal development. Neuropsychopharmacology 2004, 29(11):1988-1993.

14. Ali I, O'Brien P, Kumar G, Zheng T, Jones NC, Pinault D, French C, Morris MJ, Salzberg MR, O'Brien TJ: Enduring effects of early life stress on firing patterns of hippocampal and thalamocortical neurons in rats: implications for limbic epilepsy. PLoS One 2013, 8(6):e66962.

15. Brooks SJ, Dalvie S, Cuzen NL, Cardenas V, Fein G, Stein DJ: Childhood adversity is linked to differential brain volumes in adolescents with alcohol use disorder: a voxel-based morphometry study. Metab Brain Dis 2014, 29(2):311-321.

16. Posthuma D, De Geus E, Neale M, Pol HH, Baare W, Kahn R, Boomsma D: Multivariate genetic analysis of brain structure in an extended twin design. Behav Genet 2000, 30(4):311-319.

17. Baaré WF, Pol HEH, Boomsma DI, Posthuma D, de Geus EJ, Schnack HG, van Haren NE, van Oel CJ, Kahn RS: Quantitative genetic modeling of variation in human brain morphology. Cereb Cortex 2001, 11(9):816-824

18. Thompson PM, Cannon TD, Narr KL, van Erp T, Poutanen V, Huttunen M, Lönnqvist J, Standertskjöld-Nordenstam C, Kaprio J, Khaledy M: Genetic influences on brain structure. Nat Neurosci 2001, 4(12):1253-1258.

19. Pezawas L, Verchinski BA, Mattay VS, Callicott JH, Kolachana BS, Straub RE, Egan MF, Meyer-Lindenberg A, Weinberger DR: The brain-derived neurotrophic factor val66met polymorphism and variation in human cortical morphology. J Neurosci 2004, 24(45):10099-10102.

20. Bueller JA, Aftab M, Sen S, Gomez-Hassan D, Burmeister M, Zubieta JK: BDNF Val66Met allele is associated with reduced hippocampal volume in healthy subjects. Biol Psychiatry 2006, 59(9):812-815.

21. Liu M, Huang C, Chen M, Yang AC, Tu P, Yeh H, Hong C, Chen J, Hwang J, Lin C, Chen J: Effect of the BDNF Val66Met polymorphism on regional gray matter volumes and cognitive function in the Chinese population. Neuromolecular Med 2013, 16(1):127-136.

22. Bath KG, Lee FS: Variant BDNF (Val66Met) impact on brain structure and function. Cogn Affect Behav Neurosci 2006, 6(1):79-85.

23. Bhang S, Ahn J, Choi S: Brain-derived neurotrophic factor and serotonin transporter gene-linked promoter region genes alter serum levels of brain-derived neurotrophic factor in humans. J Affect Disord 2011, 128(3):299-304.

24. Ferrett HL, Cuzen NL, Thomas KG, Carey PD, Stein DJ, Finn PR, Tapert SF, Fein G: Characterization of South African adolescents with alcohol use disorders but without psychiatric or polysubstance comorbidity. Alcohol Clin Exp Res 2011, 35(9):1705-1715.

25. Bava S, Jacobus J, Thayer RE, Tapert SF: Longitudinal changes in white matter integrity among adolescent substance users. Alcohol Clin Exp Res 2013, 37(s1):E181-E189.

26. Churchwell JC, Carey PD, Ferrett HL, Stein DJ, Yurgelun-Todd DA: Abnormal striatal circuitry and intensified novelty seeking among adolescents who abuse methamphetamine and cannabis. Dev Neurosci 2012, 34(4):310-317.

27. Bernstein DP, Stein JA, Newcomb MD, Walker E, Pogge D, Ahluvalia T, Stokes J, Handelsman L, Medrano M, Desmond D: Development and validation of a brief screening version of the childhood trauma questionnaire. Child Abuse Negl 2003, 27(2):169-190.

28. Kaufman J, Birmaher B, Brent D, Rao U, Flynn C, Moreci P, Williamson D, Ryan $\mathrm{N}$ : Schedule for affective disorders and schizophrenia for school-age children-present and lifetime version (K-SADS-PL): initial reliability and validity data. J Am Acad Child Adolesc Psychiatry 1997, 36(7):980-988.

29. Sobell LC, Sobell MB: Timeline follow-back. In Measuring Alcohol Consumption. Edited by Anonymous Springer; 1992:41-72

30. Ashburner J, Friston KJ: Unified segmentation. Neuroimage 2005, 26(3):839-851.
31. Nordenskjöld R, Malmberg F, Larsson E, Simmons A, Brooks SJ, Lind L, Ahlström H, Johansson L, Kullberg J: Intracranial volume estimated with commonly used methods could introduce bias in studies including brain volume measurements. Neuroimage 2013, 83:355-360.

32. IBM Corp: IBM SPSS Statistics for Windows. Armonk, NY; 2012, 21.0

33. Tsai S, Liao D, Yu YW, Chen T, Wu H, Lin C, Cheng C, Hong C: A study of the association of (Val66Met) polymorphism in the brain-derived neurotrophic factor gene with alcohol dependence and extreme violence in Chinese males. Neurosci Lett 2005, 381(3):340-343.

34. Grzywacz A, Samochowiec A, Ciechanowicz A, Samochowiec J: Family-based study of brain-derived neurotrophic factor (BDNF) gene polymorphism in alcohol dependence. Pharmacol Rep 2010, 62(5):938-941.

35. Cole J, Weinberger D, Mattay $V$, Cheng X, Toga A, Thompson P, Powell Smith G, Cohen Woods S, Simmons A, McGuffin P: No effect of 5HTTLPR or BDNF Val66Met polymorphism on hippocampal morphology in major depression. Genes Brain Behav 2011, 10(7):756-764

36. Harrisberger F, Spalek K, Smieskova R, Schmidt A, Coynel D, Milnik A Fastenrath M, Freytag V, Gschwind L, Walter A: The association of the BDNF Val66Met polymorphism and the hippocampal volumes in healthy humans: a joint meta-analysis of published and new data. Neurosci Biobehav Rev 2014, 42:267-278.

37. MacDonald AW, Cohen JD, Stenger VA, Carter CS: Dissociating the role of the dorsolateral prefrontal and anterior cingulate cortex in cognitive control. Science 2000, 288(5472):1835-1838.

38. Nemoto K, Ohnishi T, Mori T, Moriguchi Y, Hashimoto R, Asada T, Kunugi H: The Val66Met polymorphism of the brain-derived neurotrophic factor gene affects age-related brain morphology. Neurosci Lett 2006, 397(1):25-29.

39. Edmiston EE, Wang F, Mazure CM, Guiney J, Sinha R, Mayes LC, Blumberg HP: Corticostriatal-limbic gray matter morphology in adolescents with self-reported exposure to childhood maltreatment. Arch Pediatr Adolesc Med 2011, 165(12):1069-1077.

40. Szeszko P, Lipsky R, Mentschel C, Robinson D, Gunduz-Bruce H, Sevy S, Ashtari M, Napolitano B, Bilder R, Kane J: Brain-derived neurotrophic factor val66met polymorphism and volume of the hippocampal formation. Mol Psychiatry 2005, 10(7):631-636.

41. Frodl T, Schule C, Schmitt G, Born C, Baghai T, Zill P, Bottlender R, Rupprecht R, Bondy B, Reiser M: Association of the brain-derived neurotrophic factor Val66Met polymorphism with reduced hippocampal volumes in major depression. Arch Gen Psychiatry 2007, 64(4):410-416.

42. Aas M, Haukvik UK, Djurovic S, Bergmann $\varnothing$, Athanasiu L, Tesli MS, Hellvin T, Steen NE, Agartz I, Lorentzen S: BDNF val66met modulates the association between childhood trauma, cognitive and brain abnormalities in psychoses. Prog Neuropsychopharmacol Biol Psychiatry 2013, 46:181-188.

43. Lipska BK, Aultman JM, Verma A, Weinberger DR, Moghaddam B: Neonatal damage of the ventral hippocampus impairs working memory in the rat. Neuropsychopharmacology 2002, 27(1):47-54.

44. Bilek E, Schafer A, Ochs E, Esslinger C, Zangl M, Plichta MM, Braun U, Kirsch $P$, Schulze TG, Rietschel M, Meyer-Lindenberg A, Tost H: Application of high-frequency repetitive transcranial magnetic stimulation to the DLPFC alters human prefrontal-hippocampal functional interaction. J Neurosci 2013, 33(16):7050-7056.

45. Burrus C: Developmental trajectories of abuse-An hypothesis for the effects of early childhood maltreatment on dorsolateral prefrontal cortical development. Med Hypotheses 2013, 81(5):826-829.

46. LaBrie JW, Migliuri S, Kenney SR, Lac A: Family history of alcohol abuse associated with problematic drinking among college students. Addict Behav 2010, 35(7):721-725. 